\title{
Ultrasonography findings of proximal-type epithelioid sarcoma of the vulva: A case report
}

\author{
YUANYUAN YUE ${ }^{1}$, YA LU $^{2}$, XIAOJUAN MA ${ }^{1}$, ZHONGPING TANG $^{3}$ and YINRONG CHENG ${ }^{1}$ \\ ${ }^{1}$ Department of Ultrasound, Chengdu First People's Hospital, Chengdu, Sichuan 610041; ${ }^{2}$ Department \\ of Pathology, West China Hospital, Sichuan University, Chengdu, Sichuan $610065 ;{ }^{3}$ Department \\ of Pathology, Chengdu First People's Hospital, Chengdu, Sichuan 610041, P.R. China
}

Received May 10, 2018; Accepted July 30, 2018

DOI: $10.3892 /$ mco.2018.1708

\begin{abstract}
Proximal-type epithelioid sarcoma (PES) of the vulva is an exceedingly rare malignant soft tissue tumor. We herein present the case of a 41-year-old female patient who presented to our hospital with complaints of a painless mass in the right mons pubis that she had first noticed 3 years prior. Ultrasonographic (US) and color Doppler ultrasonographic (CDUS) examination revealed a solid mass with well-defined, homogeneous hypoechoic structure and quite hypervascular on CDUS. The results of the immunohistochemical examination confirmed the diagnosis of vulvar PES (myxoid variant). The patient was treated with wide local excision and remained recurrence- and metastasis-free at 9 months postoperatively. Although cases of PES in the pelvic region had been previously reported, to the best of our knowledge, the US or CDUS findings of PES of the vulva have not been described to date.
\end{abstract}

\section{Introduction}

Proximal-type epithelioid sarcoma (PES) of the vulva is an exceedingly rare malignant soft tissue tumor. The first case of vulvar PES was described in 1972 (1), and <60 cases have been reported in the literature to date. Compared with other soft tissue tumors, accurate diagnosis of this neoplasm is generally challenging due to its rarity and the likelihood of misdiagnosis as a benign lesion, including Bartholin's gland cyst, Bartholin's gland abscess, inguinal or femoral hernias or other benign soft tissue tumors (2).

Several imaging modalities have been used to assess soft tissue tumors, including ultrasonography (US), computed tomography (CT) and magnetic resonance imaging (MRI),

Correspondence to: Dr Yinrong Cheng, Department of Ultrasound, Chengdu First People's Hospital, 18 Vientiane North Road, Hi-tech Zone, Chengdu, Sichuan 610041, P.R. China

E-mail: chengyr478609023@163.com

Key words: epithelioid, sarcoma, vulva, ultrasonography, color Doppler plain radiography and positron emission tomography (PET). US in particular is the most common first-line examination in vulvar lesions due to its real-time and radiation-free properties. As PES lesions may present with various and non-specific signs and symptoms, the US findings of vulvar PES may lack specificity compared with other solid masses. However, US can assess the mass size, shape, echogenicity, margin, composition and vascularity (3). Furthermore, to the best of our knowledge, US and CDUS findings of vulvar PES have not been described in the literature to date. In the present study, the case of a patient with vulvar PES is presented and the US and CDUS findings are discussed.

\section{Case report}

A 41-year old female patient presented to the Department of Ultrasonography of Chengdu First People's Hospital (Chengdu, China) in May 2017, with complaints of a painless mass in the right mons pubis that she had first noticed 3 years prior. The patient had no other significant medical history and her family history did not include malignancies in first-degree relatives. Gynecological examination of the vagina, cervix and uterus appeared normal. Inspection of the vulva revealed a mass $2 \mathrm{~cm}$ in greatest diameter in the right mons pubis. Physical examination of the mass revealed a non-tender nodule with a smooth surface and hard consistency. No other abnormalities were observed on physical examination. Gray-scale US and CDUS of the vulva were performed with a MyLab Twice ultrasound scanner (Esaote, Genova, Italy) and a 5-10 MHZ multifrequency linear-array transducer. A prominent homogenous hypoechoic and ovoid solid mass with a well-defined border, sized 27x11 mm, was detected in the subcutaneous tissue in the right mons pubis. CDUS and pulse Doppler US revealed arterial blood flow with low resistivity index and low systolic peaks $(20.9 \mathrm{~cm} / \mathrm{sec})$ in the mass (Fig. 1). The mass was clinically considered to be a benign lesion and was removed by excisional biopsy.

The initial pathology report suggested that the mass was rich in myxoid stroma. Subsequently, the surgical specimen was sent to the West China Hospital for consultation. The results of the immunohistochemical staining of the specimen were as follows: Negative for S100 protein, desmin, CD31, CD34, P40, P63, erythroblast transformation-specific-related gene, anaplastic lymphoma kinase-1, cytokeratin (CK)18, PAN-CK, 

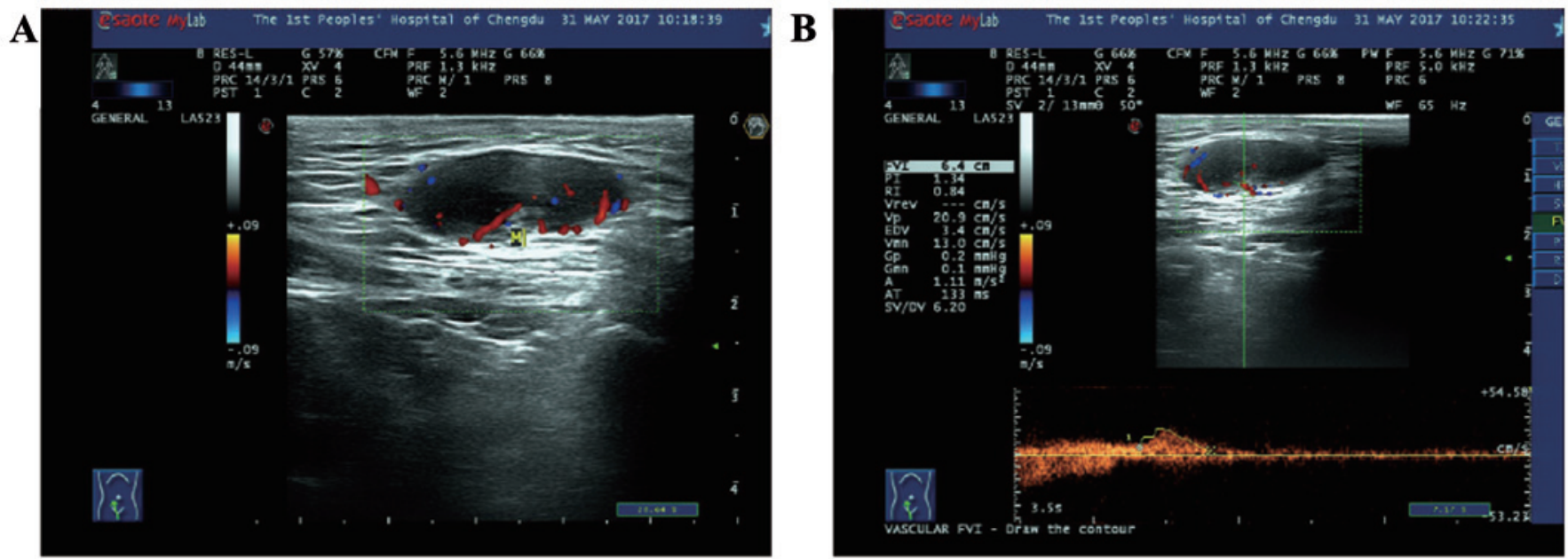

Figure 1. Real-time ultrasonogram combining Color Doppler with Pulse Doppler findings of the lesion in the subcutaneous region of the right mons pubis (A) The lesion was a homogenous hypoechoic and ovoid solid mass with a well-defined border and moderate blood flow. (B) The lesion exhibited arterial blood flow with low resistivity index and low systolic peaks $(20.9 \mathrm{~cm} / \mathrm{sec})$.
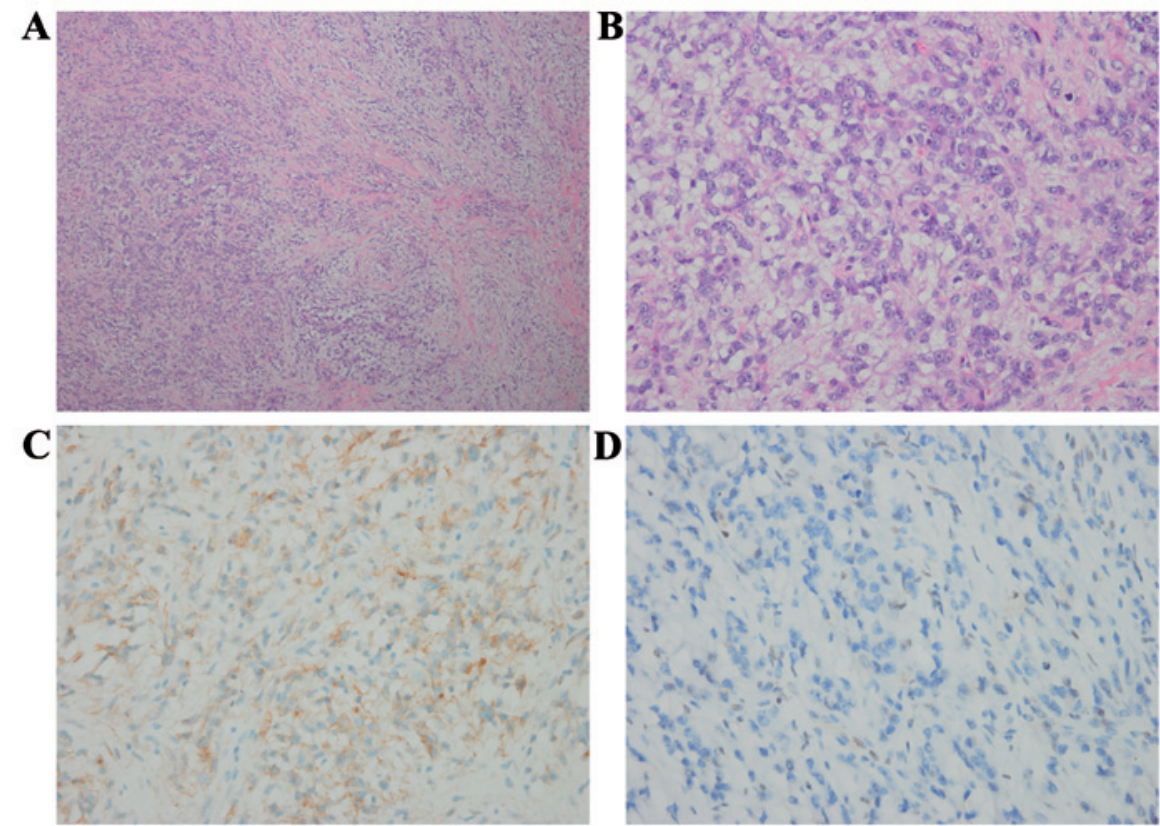

Figure 2. Hematoxylin-eosin staining and immunohistochemical expression pattern of the lesion in the mons pubis. (A) The lesion was composed of a mixed population of proliferating epithelioid and spindle cells. The transition between the two cell types is gradual and intercellular collagen deposition may be observed (magnification, x100). (B) The lesion was rich in myxoid stroma and the tumor cells displayed abundant cytoplasm, enlarged vesicular nuclei and prominent nucleoli (magnification, $\mathrm{x} 400$ ). (C) The tumor cells exhibited membranous positivity for epithelial membrane antigen (magnification, $\mathrm{x} 400$ ). (D) The tumor cells exhibited a complete loss of INI1 expression (magnification, $\mathrm{x} 400$ ).

smooth muscle actin and myogenin; the Ki-67 (MIB-1) index was positive ( $15 \%$ of the nuclei); the specimen was positive for epithelial membrane antigen (EMA) (Fig. 2). Additional INI1 staining revealed a complete loss of expression in tumor cells. These immunohistochemical results confirmed the diagnosis of vulvar PES (myxoid variant) in the right mons pubis. The patient underwent a second excision at the Sichuan Cancer Hospital. There were no residual tumor cells found in the first surgical area. The patient is currently well, without evidence of recurrence or metastasis, 9 months after the operation. During the follow-up period, the patient did not receive any adjuvant therapy.

\section{Discussion}

Epithelioid sarcoma (ES), first described by Enzinger in 1970 (4), as an independent entity, is a rare neoplasm accounting for $<1 \%$ of adult soft tissue sarcomas. Two histological variants have been identified: Classic ES (also referred to as distal-type ES) and proximal-type ES. The former generally occurs in the distal extremities (mostly the hand or wrist) of adolescents and young adults, and it may appear as solitary or multinodular lesions involving either the deep or superficial soft tissues. The latter is rarer, generally occurring in middle-aged or older patients. It often develops in the axilla and the genital 
area (groin, mons pubis, clitoris and vulva) (5). The most common initial symptom of vulvar PES is a slowly growing, relatively painless mass in the labia majora. Due to its benign appearance as a painless subcutaneous nodule, diagnosis and treatment may often be delayed (6). In the present case, PES occurred in the mons pubis as a slow-growing, painless mass. The mass was clinically considered to be a benign lesion and was removed by excisional biopsy.

US, CT and MRI, plain radiography and PET have been used to assess soft tissue tumors. As US is cost-effective, non-invasive, real-time and easy to perform, it has been used as the main first-line examination method for evaluating soft tissue tumors over several years (7). Due to the technological advances, US can be more sensitive to the detection of minute lesions. Furthermore, the combination of CDUS and spectral analysis may depict the vascularity in malignant tumors, whereas other imaging modalities cannot obtain this type of information (8). In addition, together with clinical examination, US-guided needle biopsy is a more cost-effective method for confirming the final diagnosis (3). Compared with other benign masses, soft tissue sarcomas are more likely to recur and metastasize after surgery. Fornage et al (9) reported that US may not definitively distinguish between recurrent tumor and postsurgical changes in the early postoperative period (3-6 months); however, the sensitivity and specificity of US for identification of recurrent tumors was comparable to that of routine MRI for long-term follow-up.

Immunohistochemistry is useful for differentiating epithelioid sarcomas from other tumors, including other sarcomas with epithelioid cells, malignant melanoma, metastatic carcinoma and malignant extrarenal rhabdoid tumor (MERT) (10). Generally, vulvar PES expresses epithelioid and mesenchymal markers, such as vimentin, EMA, CKs and CD34. Staining for S100 or HMB45 to exclude malignant melanoma is a particularly useful differential diagnosis for PES and MERT. The innovative molecular marker INI 1 may also be used in the differential diagnosis of soft tissue tumors (11).

Optimal treatment for PES of the vulva has not been established due to its rarity. There is no universally accepted treatment for vulvar epithelioid sarcoma (12). However, the cornerstone of initial management is wide surgical excision with an adequate margin ( $\geq 2 \mathrm{~cm}$ ) (13). The role and selection of adjuvant therapy also remain unclear due to the rarity of this disease. Adjuvant radiotherapy is advocated in high-grade tumors or cases with inadequate surgical margins (14), and also due to the high incidence of local recurrence and distant metastasis (15). However, the results of radiotherapy are controversial and show no statistically significant reduction in mortality (16). The role of chemotherapy in the adjuvant setting appears marginally effective at best for the treatment of metastatic disease (17).

In conclusion, US and CDUS may be used to evaluate vulvar PES, a rare soft tissue sarcoma, by location, echogenicity, margin, shape, composition, sound transmission and vascularity, although vulvar PES generally presents as a non-specific solid mass. Combination with other imaging modalities may aid the diagnosis. However, definite diagnosis relies on the histopathological examination.

\section{Acknowledgements}

The authors gratefully acknowledge the assistance of Dr Buyun Ma, Department of Ultrasonography, West China Hospital, Sichuan University (Chengdu, China).

\section{Funding}

No funding was received.

\section{Availability of materials and data}

Not applicable.

\section{Authors' contributions}

YY analyzed the clinical data and drafted the manuscript. YL provided the pathological results. YC provided ultrasound examination results and conducted follow-up on the patient in the outpatient clinic. ZT provided professional guidance on pathological results. XM contributed to critical review and supervised the entire study. All the authors have read and approved the final version of this manuscript.

\section{Ethics approval and consent to participate}

Not applicable.

\section{Patient consent for publication}

Written informed consent was obtained from the patient for publication of this case report and any accompanying images.

\section{Competing interests}

The authors declare no potential conflicts of interest with respect to the research, authorship and/or publication of this article.

\section{References}

1. Piver MS, Tsukada Y and Barlow J: Epithelioid sarcoma of the vulva. Obstet Gynecol 40: 839-842, 1972.

2. Altundag K, Dikbas O, Oyan B, Usubutun A and Turker A: Epithelioid sarcoma of vulva: A case report and review of the literature. Med Oncol 21: 367-372, 2004.

3. Fornage BD: Soft-tissue masses: The case for increased utilization of sonography. Ald Radiol 29: 8-22, 2000.

4. Enzinger FM: Epithelioid sarcoma. A sarcoma simulating a granulomas or a carcinom. Cancer 26: 1029-1041, 1970.

5. Moore RG, Steinhoff MM, Granai CO and Demars LR: Vulvar epithelioid sarcoma in pregnancy. Gynecol Oncol 85: 218-222, 2002.

6. Ulutin HC, Zellars RC and Frassica D: Soft tissue sarcoma of the vulva: A clinical study. Int J Gynecol Cancer 13: 528-531, 2003.

7. Choi H, Varma DG, Fornage BD, Kim EE and Johnston DA: Soft-tissue sarcoma: MR Imaging vs sonography for detection of local recurrence after surgery. AJR Am J Roentgenol 157: 353-358, 1991.

8. Belli P, Costantini M, Mirk P, Maresca G, Priolo F and Marano P Role of Color Doppler Sonography in the assessment of musculoskeletal soft tissue masses. J Ultrasound Med 19: 823-830, 2000.

9. Fornage BD: Soft tissue masses: The underutilization of sonography. Semin Musculoskelet Radiol 3: 115-134, 1999.

10. Guillou L, Wadden C, Coindre JM, Krausz T and Fletcher CD: 'Proximal-type' epithelioid sarcoma, distinctive aggressive neoplasm showing rhabdoid features. Clinico pathologic, immunohistochemical, and ultrastructural study of a series. Am J Surg Pathol 21: 130-146, 1997. 
11. Hornick JL, Dal Cin P and Fletcher CD: Loss of INI1 expression is characteristic of both conventional and proximal-type epithelioid sarcoma. Am J Surg Pathol 33: 542-550, 2009.

12. Lacazzo C, Gkegkes ID and Vrachnis N: Dilemmas in the management of patients with vulvar epithelioid sarcoma: A literature review. Eur J Obstet Gynecol Reprod Biol 176: 1-4, 2014.

13. Bos GD, Pritchard DJ, Reiman HM, Dobyns JH, Ilstrup DM and Landon GC: Epithelioid sarcoma. An analysis of fifty-one cases. J Bone Joint Surg 70: 862-870, 1988.

14. CasanovaM,FerrariA,ColliniP,BisognoG,AlaggioR,CecchettoG, Gronchi A,Meazza C, Garaventa A, Di Cataldo A, et al: Epithelioid sarcoma in children and adolescents: A report from the Italian soft tissue sarcoma committee. Cancer 106: 708-717, 2006.
15. Hasegawa T, Matsuno Y, Shimoda T, Umeda T, Yokoyama R and Hirohashi S: Proximal-type epithelioid sarcoma: A clinicopathologic study of 20 cases. Mod Pathol 14: 655-663, 2001.

16. Evans HL and Baer SC: Epithelioid sarcoma: A clinicopathologic and prognostic study of 26 cases. Semin Diagn Pathol 10: 286-291, 1993.

17. Argenta PA, Thomas S and Chura JC: Proximal-type epithelioid sarcoma vs. malignant rhabdoid tumor of the vulva: A case report, review of the literature, and an argument for consolidation. Gynecol Oncol 107: 130-135, 2007. 